\title{
Investigation of the Hierarchical Structure of Kinetic Models in Ignition Problems
}

\author{
By V. Bykov and U. Maas* \\ Institute of Technical Thermodynamics, Karlsruhe University, Kaiserstr. 12, 76128 Karlsruhe \\ Dedicated to Prof. Dr. Heinz-Georg Wagner on the occasion of his $80^{\text {th }}$ birth- \\ day.
}

(Received December 9, 2008; accepted March 8, 2009)

Chemical Kinetics / Reduction / ILDM / Ignition

In this paper a novel method for global analysis of chemical kinetic models is discussed and applied to auto ignition of high hydrocarbons. It is mainly based on the concept of decomposition of motions and follows major steps of the ILDM method. However, a very important difference of the suggested method is its ability of an explicit and global representation of the system decomposition as a standard singular perturbed system (SPS). The fact that the ILDM provides local information about the decomposition makes the ILDM quite accurate in description of the slow system dynamics, but for fast motions, which become very important in the context of ignition/extinction problems, there are up to now no reliable methods based on ILDM. Therefore, the current work is devoted to developing such a method which can be used efficiently for global analysis of the reaction mechanisms with subsequent formulation of explicit reduced models for unsteady combustion regimes like ignition processes. The suggested method is illustrated by a simple Lindemann kinetic model and then applied successfully to the auto ignition of a homogeneous n-heptane/air system.

\section{Introduction}

Mathematical modeling of combustion processes has gained importance in the recent years due to the requirement of economical use of available energy resources and reducing the overall pollution impact caused by combustion processes [1-2]. In order to further improve the technology, more detailed and reliable modeling of the combustion phenomena is needed. One of the most complicated parts of such models is obviously the chemical kinetic model.

In order to overcome problems with modeling of combustion processes and obtain better agreement with experimental data very complex and detailed mathe-

* Corresponding author. E-mail: umaas@itt.uni-karlsruhe.de 
matical models of chemical kinetics mechanisms are needed. One has to increase the complexity and the dimension of the physical model. In this way, reaction mechanisms reach in some cases more than thousand chemical species, which participate in several thousands of elementary reactions. This leads to very large dimensions of the partial differential equation systems. As a result typical mathematical models of reacting flows with detailed mechanisms [3] cannot be treated analytically and even the numerical treatment is limited to academic problems (see, for instance, results of DNS with detailed chemical kinetics models [4-5]). Accordingly, the need for reduction methods providing reduced models, which are simple (low dimensional, less complex etc.), but nevertheless, describe quantitatively the underlying combustion process, has increased [6-7]. In this respect the most important feature of chemical kinetic mechanisms is the existence of multiple time scales. Although this complicates a lot the numerical treatment of the reacting flow system due to the stiffness of the resulting mathematical model, it allows also to construct low-dimensional approximations of the detailed model describing the so-called long-term and rate limiting dynamics accurately.

There are many realizations of model reduction exploiting this property; only a very short list of methods is presented below: One of the first methods of a global model reduction are the Quasi Steady States Assumption (QSSA) and the Partial Equilibrium Assumption (PEA) [8-9], [10] (see e.g. [11-14] for more discussions and reviews). Recently, many numerical tools for model reduction have been developed: Intrinsic Low-Dimensional Manifolds (ILDMs) [15], Computational Singular Perturbation method (CSP) [16], Level Of Importance Index approach (LOI) [17], sensitivity analysis [18-19] etc. It has to be noted that the ILDM method [20] combines both the system hierarchy analysis and provides a complete method of automatic reduced model generation and its subsequent implementation into CFD codes that uses a tabulation strategy [21].

There are other recent approaches based on different minimization strategies such as Rate-Controlled Constrained Equilibrium (RCCE) [22] and Minimal Entropy Production Trajectories (MEPT) [23], and the Lumping Method (LM) [24], but they use knowledge about the hierarchical/decomposed structure that has to be provided by other methods and, consequently, require a time (human resources) consuming analysis of the hierarchy. The Method of Invariant/Integral Manifolds (MIM) [25-31] gives a mathematical basis for the most approaches listed above as well as for different optimization strategies (see e.g. [32]).

Two additional methods have to be mentioned here, namely, the flamelet [33-34] and the REDIM [35] methods which represent attempts of a systematic treatment of coupling of the chemical kinetics with the molecular transport processes [36]. In the current work, however, this coupling is not in the focus of the study. It is assumed that the transport processes have a negligible influence on the ignition process which corresponds to a pure homogeneous case with infinitely fast diffusion processes.

This study focuses mainly on developing a tool of kinetic mechanism analysis that globally explores the hierarchy (multi scales) of the system with its 
subsequent application to model reduction. By further extending a coordinatefree version of the decomposition framework and applying a linearization strategy the tool for automatic hierarchy analysis is emerged. The method is validated on a very important test case - the self ignition problem.

This paper is organized as follows: the second section will be devoted to an introduction to the mathematical modeling of chemical kinetic mechanisms within the framework of model reduction. Next, the suggested method of global system analysis will be discussed and presented by a simple example. Then, the so-called global quasi-linearization procedure (GQL) is applied to the self-ignition problem of the n-heptanes/air combustion system.

\section{Mathematical formulation and problem statement}

A typical mathematical model of a reacting system under consideration (pure homogeneous system) is presented by a system of ordinary differential equations that describes the chemical system evolution based on the mass action law. These reaction equations describe the evolution of the thermo-chemical state vector in linear vector space $\psi=\left(\psi_{1}, \ldots, \psi_{n}\right)$ in time, where $\psi_{j}$ represents such quantities as the pressure of the mixture, the enthalpy, the mass fraction/mole concentration of chemical species or their specific mole numbers. In vector notation the system of governing equations of a homogeneous system can be written as

$$
\frac{d \psi}{d t}=F(\psi), \quad \psi \in \Omega \subset R^{n} .
$$

Here the so-called chemical source term $F$ represents the chemical source. It describes the evolution of $n_{s}$ chemical species $\left(n=n_{s}+2\right.$, with two additional variables describing thermodynamic properties) participating in $n_{r}$ elementary chemical reactions. Additionally, positive invariance of the domain $\Omega$ is assumed to ensure a dissipative nature of the reaction mechanism. Now, we present a short outline of meaning of the system reduction from a mathematical point of view. In general a final aim of model reduction is a reformulation of the system (1) in an appropriate reduced form by introducing the reduced space $\theta=$ $\left(\theta_{1}, \ldots, \theta_{m}\right), m \ll n$ such that the solution of the system (1) will be accurately described by the following reduced model

$$
\frac{d \theta}{d t}=\tilde{F}(\theta), \quad \theta \in R^{m}
$$

The main question then arises: how do the systems (1) and (2) relate to each other, namely, what is the relation between the detailed $\psi=\left(\psi_{1}, \ldots, \psi_{n}\right)$ and reduced spaces $\theta=\left(\theta_{1}, \ldots, \theta_{m}\right)$. Thus, in principle, one needs to describe a relation between these spaces in order to reduce the system. If the reduced space can be represented by a low-dimensional manifold in the detailed linear vector space, which is given in explicit form

$$
M=\{\psi=\psi(\theta)\},
$$


then the system (1) is restricted/projected to the manifold $M$ yielding

$$
\frac{d \theta}{d t}=\psi_{\theta}^{+}(\theta) F(\psi(\theta)) \equiv \tilde{F}(\theta), \quad \theta \in R^{m} .
$$

The Moore-Penrose pseudo inverse $\psi_{\theta}^{+}(\theta)$ [37-38] has been used in (4), which is a well defined function unless the tangent space $T M$ given by $\psi_{\theta}(\theta)$ degenerates.

Obviously, the reduction of a given arbitrary system with prescribed dimension and accuracy is almost always impossible in principle, but fortunately, systems governed by chemical reactions have certain properties that, nevertheless, make the model reduction possible. The existence of various time scales is a known feature of chemically reacting flows, both in experiments and detailed model simulations it is observed that not the entire possible range of the thermochemical state space is typically accessed by the system states (solutions), but only a part of it. This part normally has a low-dimensional structure or can be efficiently approximated by low-dimensional manifolds.

The information about low-dimensional manifolds with certain properties (invariant, attractive, unstable, slow, fast etc.) is important for model reduction. An efficient reduced model should not only allow the estimation or approximation of the needed reduced space or manifold (the manifold containing the reduced system dynamics), but also provide us with the tool for its analysis with respect to the properties above.

To some extent, the existing methods answer the questions above, for instance, in order to obtain a low-dimensional approximation of the accessed space by the system (1) different simplifying assumptions have been introduced. The QSSA, for example, assumes that some of the species are already in steady state (their right hand sides (RHSs) equal to zero), while the PEA defines the reduced dynamics by applying the assumption that some elementary reactions are in partial equilibrium, meaning that the rates of forward and reverse reactions are equal. Other methods like the ILDM (as well as the CSP, sensitivity analysis etc.) suggest using the local information (eigenvalues and invariant eigenspaces of the Jacobi matrix of the source term), while others use the concept of invariant manifolds [30], [35].

In the following, the so called GQL method of systematic analysis of kinetic mechanisms and low-dimensional manifolds useful for model reduction is discussed and applied to formulate the reduced model to the ignition problem.

\section{Singular perturbed systems and the global quasi- linearization (GQL) method}

A simple mathematical model for the decomposition of motions leads to a very special system representation of the system (1) as a Singular Perturbed System (SPS) [39-40] 


$$
\left\{\begin{array}{l}
\frac{d U}{d t}=F_{s}(U, V) \\
\varepsilon \frac{d V}{d t}=F_{f}(U, V)
\end{array} .\right.
$$

with the so-called slow variables $U=\left(U_{1}, \ldots, U_{m_{s}}\right)$, which are assumed to change slowly compared to fast ones $V=\left(V_{1}, \ldots, V_{m_{f}}\right), m_{s}+m_{f}=n$, due to the presence of the system small parameter $0<\varepsilon \ll 1$. Then, the SPS theory can be used systematically as a natural mathematical construction and tool for the system analysis. Namely, one has the fast motions/manifolds automatically estimated by

$$
M_{f}=\left\{(U, V): U=U_{0}\right\},
$$

with the leading order of magnitude of the system small parameter $\varepsilon$ describing the difference in time scales. They obviously depend on the initial system state $\left(U_{0}, V_{0}\right)$ because the slow variables during the fast transient period are estimated to be "frozen".

The slow system manifold is then approximated (to the leading order) by

$$
M_{s}=\left\{F_{f}(U, V)=0\right\} \text {. }
$$

Note, however, that higher order approximations follow from the application of the SPS theory in a straightforward way [40]. The stability analysis of the slow manifold is related to the following eigenvalue problem for points on the manifold (7)

$$
\operatorname{Re}\left(\lambda\left(D_{V} F_{f}(U, V)\right)\right)<0,
$$

where $D_{V} F_{f}(U, V)$ is the $m_{f} \times m_{f}$ matrix of partial derivatives of the fast subsystem with respect to the fast variable $V$ and $\lambda$ are eigenvalues of this matrix (see e.g. [41] for more detailes). Accordingly, knowledge about global fast manifolds is very important in the stability analysis of a reduced model described by a slow manifold. Now, depending on the investigated dynamical regime one can use (6) or (7) as a manifold equation for the explicit form (3) and proceed with the reduction procedure (4).

In this way the problem of reduction reduces to the problem of how to identify this special representation. Clearly, by assuming only one particular asymptotical limit and performing non-dimensionalization, it might be possible (as in the simple Lindemann model below) to obtain a representation (5) of the system (1) analytically. But, in the general case, for more complex and practical systems (with large dimension, non-linearity, stiffness etc.) it is either very time/ human/computational recourses consuming or even prohibitive in some cases. At present, there is no universal method which solves this problem in a general case.

In our previous work [42], a general framework of model reduction by decomposition of motions has been suggested. It is based on a coordinate free approach. Instead of its representation by a system of ODEs, the Singular Perturbed Vector Field (SPVF) is introduced. Thus the very complex problem in a 
general case becomes treatable under some additional hypothesis about the linear structure of fast manifolds. An algorithm to construct a linear transformation to the form above has been suggested in [41]. In the current paper the algorithm is discussed using a simple model and then applied to the very important case of combustion processes (auto-ignition) in order to verify the method and explore its capabilities.

\subsection{A simple model system}

Being a very simple example, the Lindemann model illustrates the discussion above and can be considered as a motivation for the current study. Lindemann established the mechanism for unimolecular reactions (see e.g. [43]) by introducing a third body $M$ :

$$
\begin{aligned}
& A+M_{-} A^{*}+M, \\
& A^{*} \rightarrow P
\end{aligned}
$$

where $A$ is the reactant, $A^{*}$ is the excited reactant and $P$ the product. This yields the following well known mathematical model:

$$
\left\{\begin{array}{l}
\frac{d[A]}{d t}=-k_{1}^{+}[A][M]+k_{1}^{-}[M]\left[A^{*}\right] \\
\frac{d\left[A^{*}\right]}{d t}=k_{1}^{+}[A][M]-k_{1}^{-}[M]\left[A^{*}\right]-k_{2}^{+}\left[A^{*}\right] \\
\frac{d[P]}{d t}=k_{2}^{+}\left[A^{*}\right]
\end{array}\right.
$$

It can be seen from (10) that only two equations are linearly independent. This leads to a $2 \mathrm{D}$ problem. Furthermore, in order to make the system non-linear for illustration purposes we consider the third body molecules $M$ to be the same ones as $A$, i.e. $[M]=[A]$. Thus, after reformulation, simplification and nondimensionalization [41], [44] the resulting mathematical model is represented by

$$
\left\{\begin{array}{l}
\frac{d x}{d \tau}=\frac{1}{\varepsilon} y(y-x)-x \\
\frac{d y}{d \tau}=-\frac{1}{\varepsilon} y(y-x)
\end{array}\right.
$$

where $x=\left[A^{*}\right] /[A]_{0}, y=[A] /[A]_{0}$ are normalized concentrations and $\tau=k_{2}^{+} t$ dimensionless time; the additional assumptions $k_{1}^{+}=k_{1}^{-}$and the rate limiting 


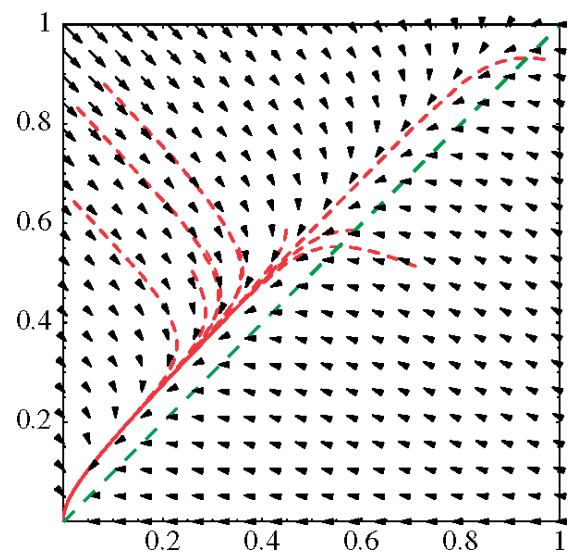

Fig. 1. Phase portrait of the Lindemann system with the vector field and trajectories at arbitrary initial conditions for $\varepsilon=0.2$. The dashed green line represents the QSSA slow curve approximation.

second step $\varepsilon=k_{2}^{+} / k_{1}^{+}<<1$ of the reaction mechanism have been made for simplicity.

\subsection{Conventional analysis}

This example can be analyzed easily by conventional asymptotic methods due to the presence of the small system parameter $0<\varepsilon \ll 1$ yielding a decomposition of motions. In this example, all methods listed in the introduction, when they are rigorously applied, are capable of a satisfactory description of the slow system manifold (up to perturbations of order $O(\varepsilon)$ in the asymptotical limit). For instance, in the QSSA any of the variables can be considered to be at the steady state: both $\frac{1}{\varepsilon} y(y-x)-x=0$ or $\frac{1}{\varepsilon} y(y-x)=0$ yield a reasonable approximation of the slow system manifold, which goes asymptotically close to the $x=y$ line (see Fig. 1, green line). The partial equilibrium assumption (PEA) corresponds to the second equation above, since the second reaction is considered as a rate limiting one, while the first reaction is at partial equilibrium along the slow manifold/curve (see Fig. 1 for illustration). Application of other methods will also differ in the first order $O(\varepsilon)$ terms one from the other. Thus, in principle, whenever the system small parameter is asymptotically small there is no problem with the slow system dynamics, the reduced space can be easily obtained, however problems begin when one needs to project an initial state of the system in the whole state space $((x, y)$ plane in this example) onto the curve representing the slow system dynamics and the system small parameter has small but finite values (see Fig. 1 for illustration). In order to make it consistent with the system dynamics the global fast motions (directions) or more specifically the way the 
system relaxes onto the slow manifold should be analyzed as well. For the model system, it can be estimated by $x+y \approx$ cons, because the following change of variables

$$
\left\{\begin{array}{l}
u=x+y \\
v=y
\end{array}\right.
$$

brings the system (11) to the standard SPS form (5)

$$
\left\{\begin{array}{l}
\frac{d u}{d t}=u-v \\
\frac{d v}{d t}=-\frac{1}{\varepsilon} v(2 v-u)
\end{array}\right.
$$

This representation and the presence of the system small parameter means that $u=x+y$ will be "frozen" (almost constant) during the fast transient period of the relaxation process towards the slow system manifold approximated by the RHS of the "fast" variable $v=y$, namely, $v(2 v-u)=y(y-x)=0$. In the considered limit, the representation (13) explicitly shows that during the fast transient period of the system behavior the concentration of the excited reactant $A^{*}$ will increase/decrease proportionally to the reactant $A$ (along the line $u=x$ $+y=$ const $)$ until the concentration approximately equalize $(x-y \approx 0)$ and then the product will be build up until both concentration will be exhausted.

\subsection{General formulation of GQL, choice of the reference set}

To start an introduction and discussion of the method of analysis suggested in this work, let us formulate the main assumption that allows using a coordinate free framework [42]. By now only the case of linear fast manifolds can be efficiently resolved by the Global Quasi-Linearization procedure [41]. It is based on linear approximations of the vector field defined by the RHS of (1), which are capable of identifying the hierarchy and globally redefine the discrepancy in time scales of the sub-processes inside a priory fixed domain in the state space. Accordingly, the core idea of the GQL is the identification of fast manifolds or subspaces in the linear vector space of the system (1).

An essence of the approach is the following procedure: in order to find the fast subspace, we suggest to identify a set of $n$ points in the defined domain in the state space $\left\{\psi^{1}, \ldots, \psi^{n}\right\} \in \Omega$, which is called a reference set and study properties of images of all vectors in the set under the vector field $F(\psi)$ (see Eq. (1) for definitions). Accordingly, if the system (1) has an internal hierarchy in the considered domain then it can be investigated and discovered by properties of linear mapping $T: \psi^{i} \rightarrow F\left(\psi^{i}\right)$, which is given by the matrix (see analysis of the Lindemann problem below)

$$
T=\left[F\left(\psi^{1}\right), \ldots, F\left(\psi^{n}\right)\right]\left[\psi^{1}, \ldots, \psi^{n}\right]^{-1}
$$

Now, if all points $\left\{\psi^{1}, \ldots, \psi^{n}\right\}$ are chosen properly (see discussion of the choice below) the linear structure of the transformation matrix $T$ should contain the 
information about the system hierarchy as well as about the fast subspace. Obviously, the crucial point of the method is associated with the choice of the reference set. In order to make a particular "representative" choice which provides us with the best fit of the vector field $F(\psi)$ by a linear transformation $T \psi$ and to overcome problems of artificial degeneration of the transformation matrix $T$ the following procedure is proposed (see e.g. [41] for more details)

- By performing a quasi-stochastic uniform distribution, an "initial set" $S_{N}$ consisting of points uniformly distributed in the domain $\Omega$ is formed

$S_{N}=\left\{\psi^{1}, \ldots, \psi^{N}\right\}, \quad N>>n$

- Then, a mean value of a vector field over the sequence $S_{N}$ is calculated

$$
\bar{F}=\frac{1}{N} \sum_{i=1}^{N} F\left(\psi^{i}\right)
$$

Now, a subset of $S_{N}$ is taken as follows

$$
S_{K}=\left\{\psi^{i} \in S_{N}:\left\|F\left(\psi^{i}\right)\right\|>\|\bar{F}\|, i=\overline{1, K}\right\}, K>>n
$$

The set $S_{K}$ is called a "separated set" and consists of points, which are "far away" from the system's slow manifold in the following sense. The norm of the vector field at these points has values above average level and can therefore safely be used to evaluate the fast subspace (consider e.g. the vector field in Fig. 1 in the vicinity of the $x=y$ line). Note that in the vicinity of the slow system manifold the system hierarchy cannot be measured, thus any subset of a length of $n$ of the control set $S_{K}$ can be used as the reference set to obtain $T$, but there is a second problem of degeneration of the chosen subset of the separated set if some of the points will be close one to another. This can lead to degeneration of the matrix $\left[\psi^{1}, \ldots, \psi^{n}\right]$ in (14) and, consequently, to a wrong decomposition. Therefore, not every subset of $S_{K}$ can be applied safely as the reference set.

- To solve the problem with degeneration we have to take a subset of vectors such that it spans the simplex of the volume which is comparable to the volume of the domain $\Omega$. Therefore, we build up the sequence of GQL approximations (14) $T_{j}, j=1, \ldots, k$ based on subsets of the control set

$$
\psi^{(i-1) n+1}, \ldots, \psi^{i n}
$$

and select only those subsets $\left\{\psi^{(j-1) n+1}, \ldots, \psi^{j n}\right\}$, which have $\operatorname{det}\left[\psi^{(j-1) n+1}, \ldots, \psi^{j n}\right]$ above the average level over all subsets

$$
\begin{aligned}
& \bar{\Delta}=\frac{1}{M} \sum_{i=1}^{M} \operatorname{det}\left[\psi^{(i-1) n+1}, \ldots, \psi^{i n}\right], \quad M \cdot n \leq K \\
& S_{C}=\left\{\begin{array}{l}
{\left[\psi^{(j-1) n+1}, \ldots, \psi^{j n}\right]:} \\
\operatorname{det}\left[\psi^{(j-1) n+1}, \ldots, \psi^{j n}\right]>\bar{\Delta}, j=1, \ldots, k
\end{array}\right\}
\end{aligned}
$$

- Now, in order to define the final GQL $T_{j}$ from the "control set" $S_{C}$ we choose the one giving the best decomposition (small parameter) for a given 
dimension $m$ of the reduced model [41]. The eigenvalues analysis of the GQL $T_{j}{ }^{*}$ answers whether there is a discrepancy in time scales, as a gap between eigenvalues $\lambda_{1}, \ldots, \lambda_{m}, \lambda_{m+1}, \ldots, \lambda_{n},\left|\lambda_{i}\right| \leq\left|\lambda_{i+1}\right|$, or not. Finally, the system small parameter can be estimated by this gap as $\varepsilon=\frac{\left|\lambda_{m}\right|}{\left|\lambda_{m+1}\right|}$.

After the reference set is properly specified the rest is simple and follows the main steps of the ILDM method [45]. The invariant subspaces $Z_{s},\left(n \times m_{s}\right)$ and $Z_{f},\left(n \times m_{f}\right)$ of the group of relatively "small" eigenvalues $\Lambda_{s},\left(m_{s} \times m_{s}\right)$ (with $\lambda_{1}, \ldots, \lambda_{m_{s}}$ on the diagonal) and of the group of "large" ones $\Lambda_{f},\left(m_{f} \times m_{f}\right)$ (with $\lambda_{m_{s}+1}, \ldots, \lambda_{n}$ on the diagonal) globally form the basis of the decomposed system as

$$
T=\left(Z_{s} Z_{f}\right)\left(\begin{array}{cc}
\Lambda_{s} & R \\
0 & \Lambda_{f}
\end{array}\right)\left(\begin{array}{l}
\tilde{Z}_{s} \\
\tilde{Z}_{f}
\end{array}\right),\left(Z_{s} Z_{f}\right)\left(\begin{array}{l}
\tilde{Z}_{s} \\
\tilde{Z}_{f}
\end{array}\right)=I_{n \times n}
$$

thus, the decomposed system in explicit form reads

$$
\left\{\begin{array} { l } 
{ U = \tilde { Z } _ { s } \psi } \\
{ V = \tilde { Z } _ { f } \psi }
\end{array} \Rightarrow \left\{\begin{array}{l}
\frac{d U}{d t}=\tilde{Z}_{s} F(U, V)=F_{s}(U, V) \\
\frac{d V}{d t}=\tilde{Z}_{f} F(U, V)=F_{f}(U, V)
\end{array} .\right.\right.
$$

\subsection{GQL analysis of the simple model}

In this subsection, in order to illustrate and give more details of the main steps of the suggested GQL method the model example (11) is considered

$$
\left\{\begin{array}{l}
\frac{d x}{d t}=f(x, y)=\frac{1}{\varepsilon} y(y-x)-x \\
\frac{d y}{d t}=g(x, y)=-\frac{1}{\varepsilon} y(y-x)
\end{array}\right.
$$

Now, to implement the method we should define two points (reference set) [41] $\left(x_{i}, y_{i}\right)^{T}, i=1,2$ in $\Omega=[0,1]^{2}$ and then find a linear transformation that transfers these points into their images under the vector field given by the system's RHS:

$$
\begin{gathered}
T:\left(\begin{array}{l}
x_{1} \\
y_{1}
\end{array}\right) \rightarrow\left(\begin{array}{l}
f\left(x_{1}, y_{1}\right) \\
g\left(x_{1}, y_{1}\right)
\end{array}\right) \\
T:\left(\begin{array}{l}
x_{2} \\
y_{2}
\end{array}\right) \rightarrow\left(\begin{array}{l}
f\left(x_{2}, y_{2}\right) \\
g\left(x_{2}, y_{2}\right)
\end{array}\right)
\end{gathered}
$$

This transformation is given by the following formula

$$
T=\left(\begin{array}{l}
f\left(x_{1}, y_{1}\right) f\left(x_{2}, y_{2}\right) \\
g\left(x_{1}, y_{1}\right) g\left(x_{2}, y_{2}\right)
\end{array}\right)\left(\begin{array}{l}
x_{1} x_{2} \\
y_{1} y_{2}
\end{array}\right)^{-1}
$$

Hence, we obtain the linear map $T$ which coincides (gives same result) with the non linear map $F$ on the reference set. It is easy to check for a chosen $\psi^{i}=$ $\left(x_{i}, y_{i}\right)^{\mathrm{T}}, i=1,2$ that 


$$
\begin{aligned}
T\left(\begin{array}{l}
x_{\mathrm{i}} \\
y_{\mathrm{i}}
\end{array}\right) & =\left(\begin{array}{ll}
\frac{1}{\varepsilon} y_{1}\left(y_{1}-x_{1}\right)-x_{1} & \frac{1}{\varepsilon} y_{2}\left(y_{2}-x_{2}\right)-x_{2} \\
-\frac{1}{\varepsilon} y_{1}\left(y_{1}-x_{1}\right) & -\frac{1}{\varepsilon} y_{2}\left(y_{2}-x_{2}\right)
\end{array}\right)\left(\begin{array}{l}
x_{1} x_{2} \\
y_{1} y_{2}
\end{array}\right)^{-1}\left(\begin{array}{l}
x_{\mathrm{i}} \\
y_{\mathrm{i}}
\end{array}\right) \\
& \equiv\left(\begin{array}{l}
\frac{1}{\varepsilon} y_{i}\left(y_{i}-x_{i}\right)-x_{i} \\
-\frac{1}{\varepsilon} y_{i}\left(y_{i}-x_{i}\right)
\end{array}\right)=F\left(\begin{array}{l}
x_{\mathrm{i}} \\
y_{\mathrm{i}}
\end{array}\right)
\end{aligned}
$$

The result of the GQL analysis implementation is summarized in Fig. 2, where two cases of the reference set are presented. The left figure shows the resolved decomposed structure based on an arbitrary choice of the reference set $\psi^{1}=(0.6,0.2)^{\mathrm{T}}$ and $\psi^{2}=(0.1,0.7)^{\mathrm{T}}$ providing with $\widetilde{Z}_{f}=(-0.570 .87)$ and $\widetilde{Z}_{s}=$ $(0.660 .81)$ which were chosen to satisfy partial requirements of the choice procedure above (they are "far away" from the slow curve and span the volume which is compared to the domain), but certainly they are not optimal. The right figure represents the result based on the accurate choice $\psi^{1}=(0.006,0.98)^{\mathrm{T}} \psi^{2}=(0.447$, $0.843)^{\mathrm{T}}$ of the reference sequence as described in subsection 3.2 with $\widetilde{Z}_{f}=$ $(-0.770 .62)$ and $\widetilde{Z}_{s}(-0.67-0.74)$ correspondingly.

The fast manifolds/directions are calculated by the corresponding invariant subspaces (15) by $u=\widetilde{Z}_{s} \psi_{0}=$ const, whilst the system slow manifold/curve is approximated by $F_{f}(u, v)=\widetilde{Z}_{f} F\left(\left(Z_{s} Z_{f}\right)(\stackrel{u}{v})\right)=0$. This can be seen in Fig. 2 where both realizations are shown in the original coordinates $\psi=(x, y)$ by black and blue lines correspondingly. There are three main observations we would like to underline here. It can be seen clearly that the GQL method represents the real system behaviour much better (blue curve) than the standard QSSA assumption (green curve). The first observation concerns the accuracy of the result given by the arbitrary choice. The sine of the angle between $\widetilde{Z}_{s}$ for these different choices equals $\sim 0.08$ that means the deviation of fast manifolds is of order $O(\varepsilon)(\varepsilon=$ 0.2 ). By comparing intersection points of the blue slow lines with the line $y=1$ in Fig. 2 one can see as well that the deviations of the slow curves are of the same order.

The second observation can be made concerning the accuracy of the slow manifold near the origin, which in this case is a singular point of a parabolic type (one eigenvalue of the Jacobi matrix is zero). In this case even the standard expansions of the SPS theory fail to approximate the slow manifold in the vicinity of such points. The GQL method overcomes this difficulty and estimates the slow manifold quite reasonably near the origin (see Fig. 2). Finally, the suggested form of the GQL (13) allows to use the algorithm to the system in dimensional form because it is scaling invariant, and, therefore proper non-dimensionalization and normalization problems are automatically overcome [41]. 

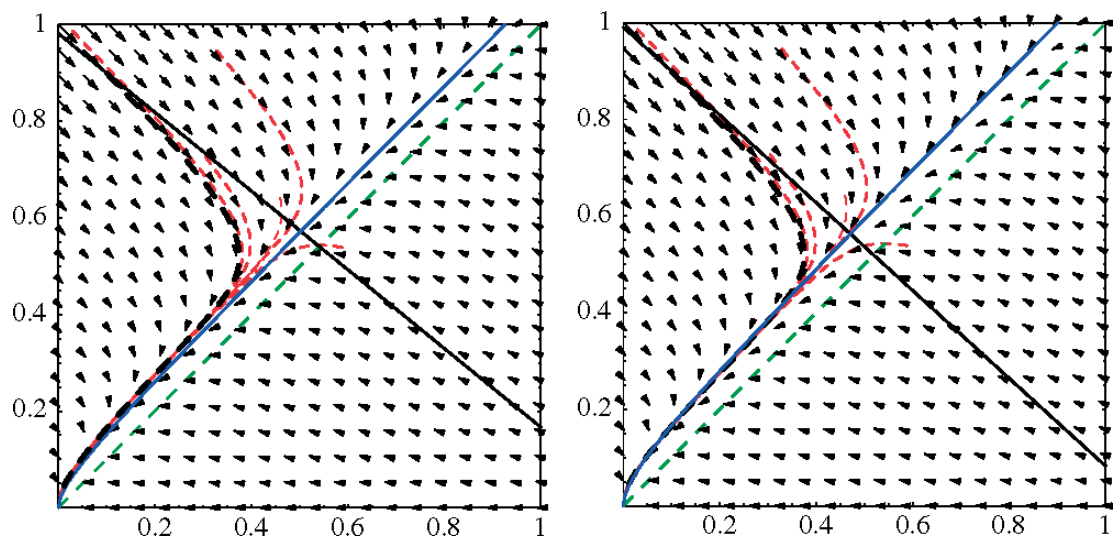

Fig. 2. The left figure shows the results of the GQL analysis for the arbitrary choice, the right figure corresponds to the accurate choice of the reference points. Black solid lines are fast system directions starting at $(0,1)$; blue curves show the GQL zero's order approximations of the system slow manifold; green lines indicate the QSSA slow curve.

\section{4. n-heptane auto-ignition problem}

\subsection{Problem definition}

In this section the method of global analysis outlined in the previous sections is applied to the homogenous auto-ignition in the n-heptane/air system. The elementary kinetic mechanism of this system is relatively well studied (see e.g. [46-49] for more references), and moreover, the complex two stage ignition of a stoichiometric mixture represents a quite interesting and challenging task for any reduction procedure.

Two stage auto-ignition occurs in the so-called Negative Temperature Coefficient (NTC) regime due to the fact that the oxidation process slows down while the temperature slightly increases (see Fig. 3). In the second stage, the formation of $\mathrm{H}_{2} \mathrm{O}_{2}$ becomes more important, which then slowly dissociates to $\mathrm{OH}$ radicals [46] leading to the second stage of thermal runway. For the analysis we have used the $n_{r}=56$ step skeletal mechanism with $n_{s}=35$ species of [47]. It is comparatively small but, nevertheless, until the second stage of the ignition it describes the kinetics qualitatively and quantitatively reasonably well (see e.g. [47] for detail and Fig. 3 for illustration). Figure 4 shows further comparisons of the chosen kinetic mechanism with the others given by LLNL [48] and Golovichev [49] of $n_{s}=159$ and $n_{s}=57$ species correspondingly. Note that although 


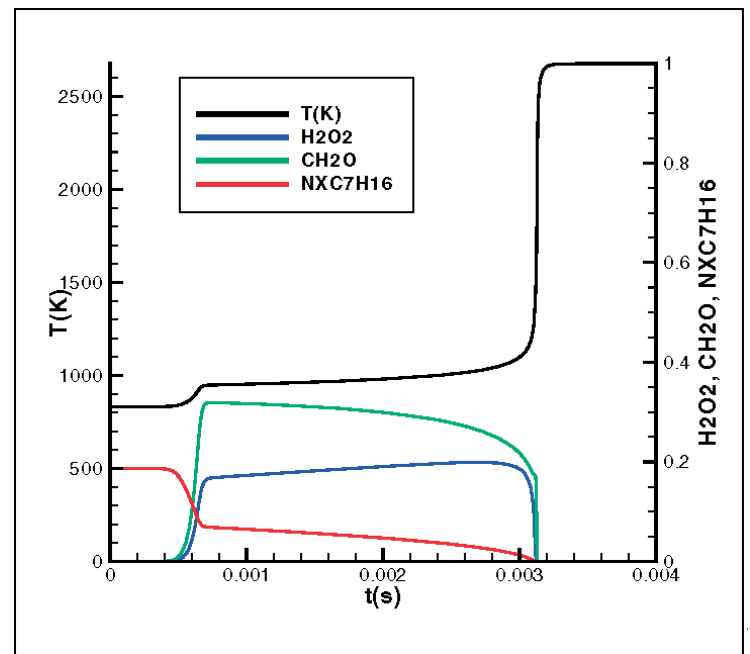

Fig. 3. Time histories of some species and temperature of n-heptane self ignition at initial temperature of $T_{0}=833 \mathrm{~K}$ and pressure $13.5 \times 10^{5} \mathrm{~Pa}$.

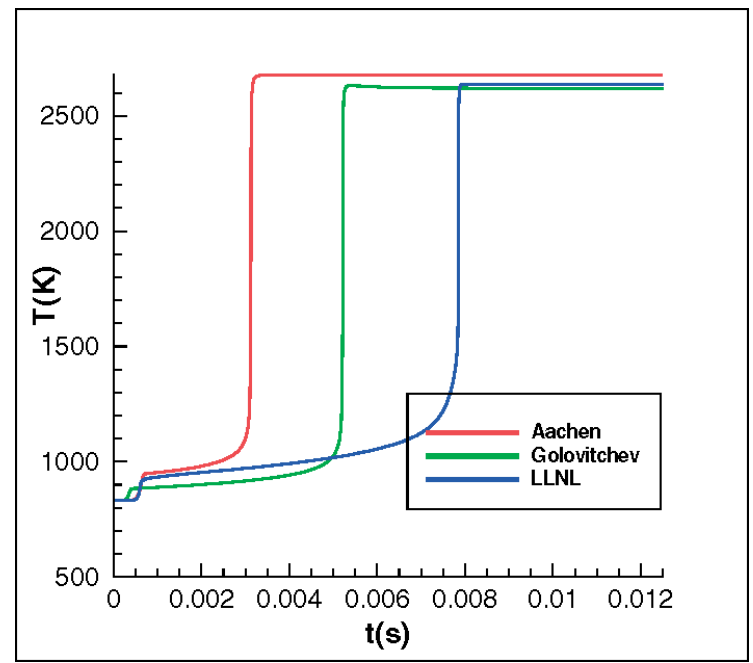

Fig. 4. Temperature time histories of different detailed mechanisms of n-heptane self ignition at initial temperature of $T_{0}=833 \mathrm{~K}$ and constant pressure $13.5 \times 10^{5} \mathrm{~Pa}$.

all these mechanisms are well accepted they predict ignition delay times varying by a factor of 3 . 


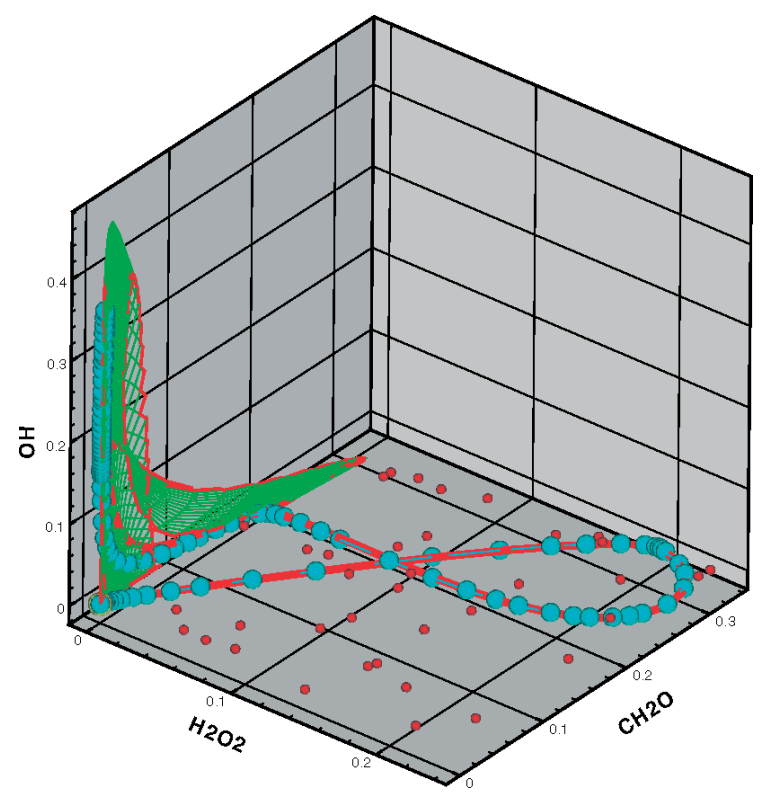

Fig. 5. State space projection onto $\mathrm{H}_{2} \mathrm{O}_{2}-\mathrm{CH}_{2} \mathrm{O}-\mathrm{OH}$ space with detailed system solution trajectory shown by the red line, $2 \mathrm{D} \mathrm{GQL}$ slow manifold is the green mesh, red cubes are the reference set, cyan line with spheres is the reduced model solution trajectory.

\subsection{GQL implementation}

Now, we use the Global Quasi Linearization (GQL) procedure to set up a decomposed system (15) for the auto-ignition problem above. The first difficulty with the implementation is a definition of a positively invariant domain (domain of interest) in the state/composition space. To overcome this problem, numerical solutions using the detailed mechanism have been used similar as suggested in [41].

The domain of interest has been defined by a system trajectory yielding the maximum values for the species molar numbers over this trajectory for all species. The detailed system solution is analyzed until the middle stage of the second delay time in order to insure that the domain of interest will cover the essential part of the ignition and will not include the slow processes close to the equilibrium point. In this respect the domain definition problem becomes a crucial point because the information on the vector field near the equilibrium, where the major products have been already build up, is not important for the ignition stage, therefore the random points are selected from the domain of the state space close to the trajectory during the ignition phase (see Fig. 5 for illustration).

Another known problem, that has to be resolved during the implementation, is the determination of the reduced system's dimension. In order to accurately 
predict the delay time one needs to keep the overall dimension of the reduced model relatively high in comparison, for instance, to a flame propagation problem. This is because the flame propagation process is mainly controlled by a few rate limiting slow reactions, which are described reasonably well by relatively low dimensional slow manifolds [45]. At the same time to describe the ignition process important fast modes have to be included in the reduced model as well. This feature is shown in the Fig. 5, where the 2D GQL approximation of the slow system manifold is presented together with the detailed and the reduced model solutions trajectories as well as with the reference set used for the GQL analysis. One can see that during the first ignition and the transient second delay period where $\mathrm{H}_{2} \mathrm{O}_{2}$ and $\mathrm{CH}_{2} \mathrm{O}$ are being formed, the system solution deviates significantly from the 2D slow manifold. However, close to equilibrium the 2D manifold represents the system quite well.

The steps of the GQL analysis are the following:

- Definition of the domain of interest $\Omega$ by detailed system trajectory analysis;

- Choice of the optimal reference set for the GQL in $\Omega$;

- Eigenvalues and eigenspaces analysis of the GQL approximation of the vector field;

- Explicit decomposition of the system by using invariant subspaces;

- Integration of the system confined to the GQL approximation of the slow system manifold yielding the reduced model;

- Comparison to the detailed system solutions for different initial temperatures and pressures for validation purpose.

\subsection{Results and discussion}

In this section, important details of the implementation scheme given above together with the outcome of the GQL analysis are presented and discussed. Ignition delay times of the original and reduced models will be compared for verification of the selected model. Different definitions of the domain have been tried, but the most simple and obvious turned out to be the following

$$
\begin{aligned}
& \psi_{i}^{\max }=\max _{0 \leq t \leq t^{*}}\left(\psi_{i}(t)\right), \\
& \Omega=\left\{\psi: 0 \leq \psi_{i} \leq \psi_{i}^{\max }, i=1, \ldots, n_{s}\right\}
\end{aligned}
$$

here $\psi_{i}(t)$ are components of a typical detailed reference solution (except those whose values are conserved) and $t^{*}$ is the time corresponding to the half of the second transient period of the overall delay (see Fig. 3). Then, an accurate choice of the reference set has been performed as described in [41] yielding the reduced model dimension as $m_{s}=20-n_{c}=14$, where $n_{c}=6$ is a number of the conserved quantities, which corresponds to the $m_{f}=17$ dimensional fast manifolds structure ( $n=m_{s}+m_{f}+n_{c}=37$ ). The gap between the eigenvalues of the GQL based on the reference set defines the system small parameter as $\varepsilon=3.42 \times 10^{-2}$. Next, the invariant subspaces of the GQL define the decomposition (16). Now, in order 

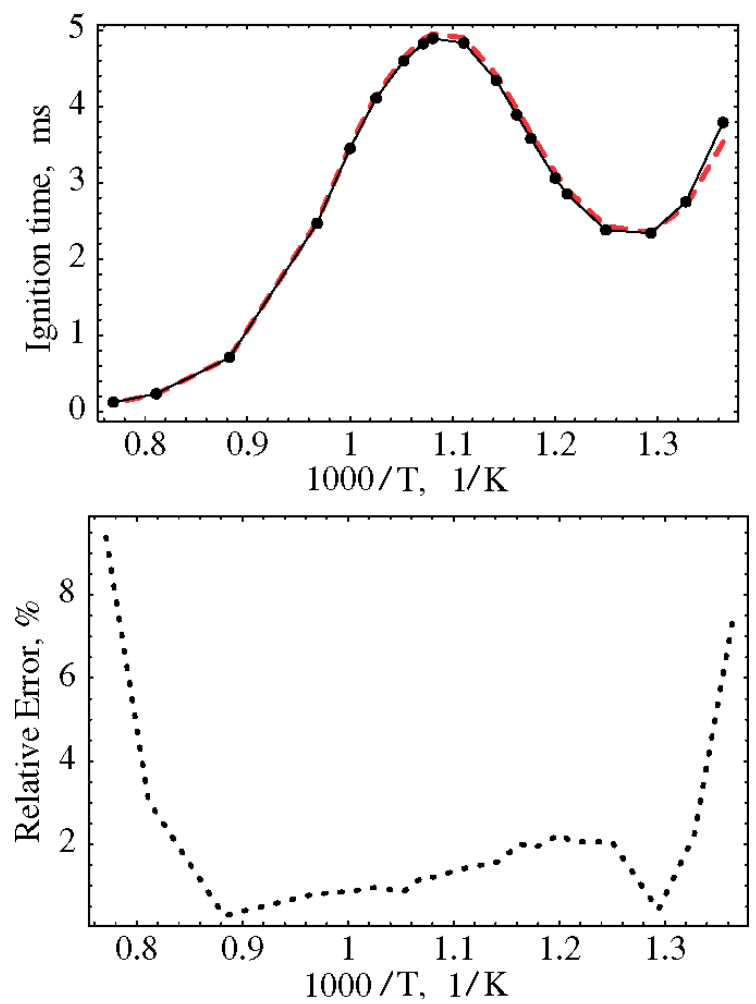

Fig. 6. Temperature dependence of the ignition delay time and the relative error. Black lines with filled circles are the reduced model delay time prediction; red dashed lines are the detailed model results.

to compare the detailed and reduced models the system has been integrated on the GQL approximation $F_{f}(U, V)=0$ of the $\mathrm{m}_{\mathrm{s}}$-dimensional slow manifold according to

$$
\left\{\begin{array}{l}
\frac{d U}{d t}=F_{s}(U, V) \\
0=F_{f}(U, V)
\end{array} .\right.
$$

In the following results of the solution of two systems (1) and (21) are compared, where the second fully corresponds to the integration of the system on the 14-dimensional slow manifold (excluding the conserved quantities i.e. it is considered in the reacting space only) which defines a 14-dimensional reduced model for the ignition process. Figure 6 compares the two delay times for initial relatively low temperatures ranging from $800 \mathrm{~K}$ to $1300 \mathrm{~K}$. It shows relative errors of the reduced model estimations as well. One sees that there are some deviations for high temperatures and pressures because the delay time becomes 

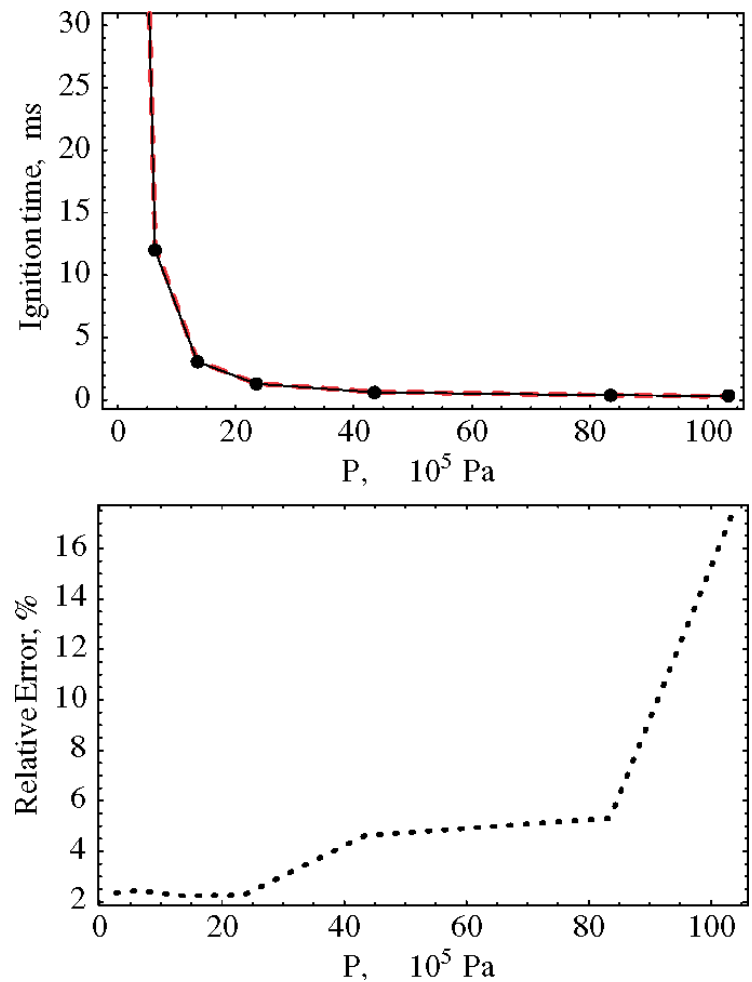

Fig. 7. Shows pressure dependence of the ignition delay time with the relative error. Black lines with filled circles are the reduced model delay time prediction; red dashed lines are the detailed model results.

very small, but they are still within an acceptable level of accuracy. It is interesting that the relative error in most of the range is less than a few percent and smaller than the differences in the results obtained from different mechanisms shown in Fig. 4.

Finally, Fig. 7 summarizes the performance of the reduced model with respect to pressure variations. Once again one sees the very high accuracy of the reduced model even for considerably high pressures, especially in the case there was no optimization of the method implemented for different pressures.

\section{Conclusions}

An efficient algorithm of the global analysis of the chemical kinetic mechanisms has been presented and applied to the problem of self ignition. It is based on the natural assumption of the decomposition of motions. It decouples the fast mo- 
tions/processes and as a result reduces the system's dimension and stiffness of such models making them treatable numerically even for complex reacting flow problems. A method which realizes this strategy has been presented and applied to an auto-ignition process. The main feature of the novel approach compared to other approaches is its global character and the capability of approximating not only the slow system manifolds (used to reduce the system), but the explicit decomposition and the fast manifolds as well. The GQL and its detailed implementation scheme is presented and fully illustrated by the planar model of the Lindeman system.

The skeletal chemical reaction mechanism (35 species, 56 reactions) for $n-$ heptane/air auto-ignition problem is considered for rigorous verification. The reduced model is formulated and studied on a basis of special system representation as a standard SPS system. The global analysis yields the 14-dimensional reduced model. The simulations of the ignition times are in very good agreement with those obtained with the detailed mechanism.

\section{Acknowledgement}

This research was supported by the Deutsche Forschungsgemeinschaft (DFG) within the (SFB 606) and (SPP 1276 - MetStöm).

\section{References}

1. An Energy Policy for Europe, Communication from the Commission to the European Council and the European Parliament COM (2007).

2. http://ec.europa.eu/energy/energy_policy/doc/01_energy_policy_for_europe_en.pdf.

3. J. Warnatz, U. Maas and R. Dibble, Combustion. 4 edn. Springer-Verlag, Berlin Heidelberg (2004).

4. M. Baum, Direct Numerical Simulation - A Tool to Study Turbulent Reacting Flows. Annual Reviews of Computational Physics, D. Staufer (ed.), Vol. 5, World Scientific Publishing Company (1997).

5. U. Maas and D. Th'evenin, Proc. Comb. Inst. 27 (1998) 1183.

6. N. Peters and B. Rogg, Reduced Kinetics Mechanisms for Application in Combustion Systems. Springer, Berlin (1993).

7. A. S. Tomlin, T. Tur' anyi and M. J. Pilling, Mathematical Tools for the Construction, Investigation and Reduction of Combustion Mechanisms. Comprehensive Chemical Kinetics 35: Low-temperature Combustion and Autoignition, Elsevier, Amsterdam (1997).

8. M. Bodenstein, S. C. Lind, Z. Phys. Chem. 57 (1906) 168.

9. J. R. Bowen, A. Acrivos, and A. K. Oppenheim, Chem. Eng. Sci. 18 (1963) 177.

10. F. A. Williams, Combustion Theory, The Fundamental Theory of Chemically Reacting Systems. $2^{\text {nd }}$ ed. Benjamin/Cummings, Menlo Park, California (1985).

11. S. H. Lam, Comb. Sci. Tech. 179 (2007) 767.

12. A. N. Gorban, N. Kazantzis, Y.G. Kevrekidis, H.C. Ottinger and C. Theodoropoulos, Model Reduction and Coarse-Graining Approaches for Multiscale Phenomena. Springer, Berlin (2006).

13. J. F. Griffiths, Prog. Energy Combust. Sci. 21 (1995) 25.

14. M. S. Okino and M. L. Mavrovouniotis, Chem. Rev. 98(2) (1998) 391. 
15. U. Maas and S. B. Pope, Combust. Flame 88 (1992) 239.

16. S. H. Lam and D. M. Goussis, Int. J. Chem. Kinet. 26 (1994) 461.

17. T. Løvås, P. Amnéus, F. Mauss and E. Mastorakos, Proc. Comb. Inst. 29 (2002) 1387.

18. H. Rabitz, M. Kramer and D Dacol, Rev. Phys. Chem. 34 (1983) 419.

19. T. Tur'anyi, J. Math. Chem., 5 (1990) 293.

20. U. Maas and S. B. Pope, Proc. Comb. Inst. 24 (1992) 103.

21. T. Blasenbrey, D. Schmidt, and U. Maas, Proc. Comb. Inst. 28 (1998) 505.

22. W. P. Jones and S. Rigopoulos, Comb. Flame 142 (2004) 223.

23. D. Lebiedz, J. Chem. Phys. 120(5) (2004) 6890.

24. G. Li and H. Rabitz, Chem. Eng. Sci. 46 (1991) 95.

25. S.J. Fraser, J. Chem. Phys. 88 (1988) 4732.

26. M. R. Roussel and S. J. Fraser, J. Chem. Phys. 93 (1990) 1072.

27. M. J. Davis and R. T. Skodje, J. Chem. Phys. 111 (1999) 859.

28. J. Nafe and U. Maas, Comb. Theory and Modell. 6(4) (2002) 697.

29. A. N. Gorban, I. V. Karlin, Chem. Eng. Sci. 58 (2003) 4751.

30. A.N. Gorban, I.V. Karlin, Invariant Manifolds for Physical and Chemical Kinetics. Lect. Notes Phys. 660, Springer, Berlin, Heidelberg (2005).

31. A. N. Gorban, I. V. Karlin, A. Y. Zinovyev, Phys. Rep. 396 (2004) 197.

32. L. Petzold and W. Zhu, AIChE. J. 45 (1999) 869.

33. N. Peteres, Proc. Comb, Inst. 21 (1986) 1231.

34. J. A. van Oijen and L. P. H. de Goey, Combust. Sci. Technol. 161 (2000) 113.

35. V. Bykov, U. Maas, Comb. Theory and Model. 11(6) (2007).

36. V. Bykov, U. Maas, Proc. Comb. Inst. 32 (2009) in press.

37. E. H. Moore, Bulletin of the American Mathematical Society 26 (1920) 394.

38. R. Penrose, Proceedings of the Cambridge Philosophical Society 51 (1955) 406.

39. N. Fenichel, J. Diff. Equ. 31 (1979) 53.

40. V. Gol'dshtein and V. Sobolev, Qualitative Analysis of Singularly Perturbed Systems of Chemical Kinetics, in Singularity Theory and some Problems of Functional Analysis. American Mathematical Society, Translations, ed. S.G. Gindikin, 153(2) (1992) 73.

41. V. Bykov, V. Gol'dshtein, U Maas, Comb. Theory and Model. 12(2) (2008) 389.

42. V. Bykov, I. Goldfarb and V. Gol'dshtein, J. Phys.: Conf. Ser. 55 (2006) 22.

43. F. A. Lindemann, Trans. Faraday Soc. 17 (1922) 598.

44. W. Richardson, L. Volk, K.H. Lau, S.H. Lin and H. Eyring, Proc. Nat. Acad. Sci. USA 70(5) (1973) 1588.

45. U. Maas, Comput. Visual. Sci. 1 (1998) 69.

46. N. Peters, G. Paczko, R. Seiser, K. Seshadri, Comb. Flame 128 (2002) 38.

47. N. Peters, Multiscale Combustion and Turbulence. Proc Comb. Inst. 32 (2009) in press.

48. http://www.llnl.gov.

49. V. Golovitchev available at: <http://www.tfd.chalmers.se/ val eri/ MECH.html>. 
